\title{
IC9-GD2-CD28-OX40-expressing T Lymphocytes
}

National Cancer Institute

\section{Source}

National Cancer Institute. iC9-GD2-CD28-OX40-expressing T Lymphocytes. NCI

Thesaurus. Code C106123.

Modified T-lymphocytes expressing a 3rd generation chimeric antigen receptor (CAR) specific for the disialog ang lioside GD2, which contains the CD3zeta chain, the signaling domains of the co-stimulatory molecules CD28 and CD134 (OX-40) and the suicide gene inducible caspase 9 (iCasp9), with potential immunomodulating and antineoplastic activities. Upon administration, iC9-GD2-CD28-OX40-expressing T lymphocytes target the GD2 antigen on tumor cells, thereby providing selective toxicity towards GD2expressing tumor cells. iCasp9 consists of a human FK506 drug-binding domain with an F36V mutation (FKBP12-F36V) linked to human caspase 9. If the administered T cells lead to unacceptable side effects, the chemical homodimerizer AP1903 can be administered, which binds to the drug binding FKBP12-F36V domain and activates caspase 9, resulting in the apoptosis of the administered T-cells. The tumor associated antigen GD2 is overexpressed on the surface of almost all tumors of neuroectodermal origin. OX40 and CD28, both T-cell surface-associated co-stimulatory molecules, are required for full Tcell activation. 